Jane Bell', Lauren Alexander ${ }^{1}$, Jane Carson ${ }^{1}$, Amanda Crossan², John McCaughan ${ }^{3}$, Hazel Mills', Damian O'Neill' , John E. Moore ${ }^{2,4}$ and B. Cherie Millar ${ }^{2,4}$, on behalf of the Northern Ireland Working Group on Nebuliser Care and Hygiene in Cystic Fibrosis

患bcmillar@niphl.dnet.co.uk

@NebsNi

in.

www.linkedin.com/in/ni-nebs-9b71381b1

\title{
Review
}

\section{Nebuliser hygiene in cystic fibrosis: evidence-based recommendations}

Nebulised therapies are extensively used in the daily therapeutic management of cystic fibrosis both for mucociliary clearance and for the management of chronic infections. Extensive developments have been made in relation to nebulised drug delivery mechanisms and drug formulations, and guidelines have been prepared that have addressed the appropriate use of such therapies. However, due to these developments, a plethora of nebuliser devices and drug chambers exist, and frequently, the limited guidance provided in relation to nebuliser hygiene is to follow manufacturers' instructions. Such instructions are inconsistent and at times confusing, translating to an increase in the burden associated with nebuliser maintenance. An evidence-based universal guideline relating to nebuliser care and hygiene is urgently required that is applicable to both at-home use and inpatient use. This article reviews the scientific literature in order to propose an evidence-based approach to nebuliser hygiene to ensure optimum drug delivery, and infection prevention and control.

\section{Educational aims}

- To understand the reasons why nebuliser hygiene is important.

- To give an overview of the current nebuliser care instructions that have been described by manufacturers, societies and the scientific literature.

- To outline the current nebuliser hygiene practices used by persons with cystic fibrosis in the home and hospital settings.

- To highlight areas that need further evaluation to promote optimum nebuliser care.

- To establish an evidence-based guideline for nebuliser hygiene in relation to cystic fibrosis.

@ERSpublications

Current nebuliser hygiene practice is varied and challenging at home and in hospital. This review of the scientific literature proposes an evidence-based approach to nebuliser hygiene to ensure optimum drug delivery and infection prevention and control. https://bit.ly/3b9jGee
Cite as: Bell J, Alexander L, Carson J, et al. Nebuliser hygiene in cystic fibrosis: evidence-based recommendations. Breathe 2020; 16: 190328.

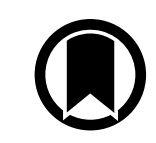

CrossMark

(c) ERS 2020 
Choice of nebuliser device used in the therapy of patients with cystic fibrosis (CF) is dictated by the presence of clinical efficacy studies, device specifications, drug dosage and individual patient characteristics, meaning that not all patients can use the same nebuliser parts. Indeed, several different nebulisers may be required for use over time in the case of a single patient [1, 2]. Due to the variety of nebulisers used, the manufacturers' advice is differing and often conflicting. Both the European Respiratory Society "Guidelines on the use of nebulisers" [3] and the British Thoracic Society "Guidelines for non-CF bronchiectasis" [4] recognise that the ideal standards and methods have not been established, and that a universal code of practice should be produced for the maintenance of nebuliser equipment. Although this code of best practice has not yet been generated, following manufacturers' instructions, as well as local infection and prevention control policies, are generally recommended.

\section{Sources of information}

A search of peer-reviewed scientific literature was performed. PubMed, Web of Science and the Cochrane Library were searched for studies evaluating microbial contamination, nebuliser hygiene, cleaning and disinfection methods. Searches were performed and all articles published since 1985 in English were initially considered (figure S1). Guidelines published by cystic fibrosis, lung and respiratory societies were also examined as well as manufacturers' guidelines of the main nebuliser units and drug chambers used within the CF community in the UK (tables S1 and S2). The articles selected for inclusion of this review article specifically contained evidence to reliably inform the recommendations contained in this article, relating to nebuliser hygiene.

\section{Definitions of key terms}

A table of key terms and definitions is detailed in table 1, explaining the various terminologies used throughout this review.

\section{Why is there a need to clean and disinfect nebulisers?}

\section{Nebuliser performance}

Nebuliser maintenance plays an important role in terms of performance, particularly in the case of electronic vibrating mesh nebulisers such as the e-Flow Rapid (Pari, Byfleet, UK). Such nebulisers offer several advantages particularly in terms of improved delivery of specific drug doses at target specific sites and a reduction in nebulisation time with less attributed mechanical noise. It must be realised, however, that since such nebulisers possess a mesh containing 4000 identical holes (2-4 $\mu \mathrm{m}$ in diameter), there is great potential for these holes to become blocked or plugged if not cleaned regularly. This, in turn, may result in the need for increased nebulisation time and also a lowered quality of aerosolised drug delivery [5, 6].

It has been reported that although patients are familiar with the importance of nebuliser care, often, the mesh component is never cleaned nor is an appropriate method of disinfection chosen, which in turn will have damaging effects on the mesh [5, 7]. Decreased nebuliser output, increased nebulisation duration and nebulisers prematurely switching off have been reported in a number of studies examining e-Flow Rapid nebuliser evaluation delivering tobramycin over a 6-month period [7, 8]. Due to the fact that the manufacturer's MeshCare system, which involves a reverse rinse of the nebuliser mesh with isotonic saline ( $5 \mathrm{~mL}$ for $20 \mathrm{~min}$ ) by means of a bespoke small shower head significantly improves nebuliser performance, it is advised to use this system on a monthly basis and particularly when the duration of nebulisation time is increased [8].

\section{Microbial contamination}

It has been widely reported that respiratory therapy equipment can become contaminated by Gramnegative bacteria and several outbreaks have been reported with fatal consequences following contamination of disinfected nebulisers with Pseudomonas aeruginosa in patients with COPD [9]. Nebuliser maintenance, cleaning and disinfection are of utmost importance as nebuliser parts, namely the compressor tubing, drug chamber, T-piece and mouthpiece, may be contaminated with both bacteria and fungi (table 2 ). The diversity of bacteria and fungi that have been found on CF nebulisers is extensive, whereby a total of 35 bacterial genera have been isolated, including 24 genera belonging to Gram-negative bacteria, 10 genera belonging to Gram-positive bacteria and a single genus, Paenibacillus, that is Gram variable. Of concern is the fact that it has been noted that CF patients' devices were positive for clinically significant organisms, namely Burkholderia cenocepacia, Stenotrophomonas maltophilia and $P$. aeruginosa. Regarding diversity of yeasts and filamentous fungi contaminating CF nebulisers, two genera belonging to the yeasts and 14 genera belonging to the filamentous fungi have been reported. Yeasts and moulds have been reported in high prevalence $(57.7 \%)$ in nebuliser parts from CF patients even after washing and drying [12, 14].

Most recently, a study from Brazil examined microbial contamination of Pari nebulisers amongst 77 CF patients across seven CF centres in Brazil [14]. All patients within the study were chronically colonised with $P$. aeruginosa. The study found a variety of pathogenic organisms following 
Table 1 Key terms and definitions

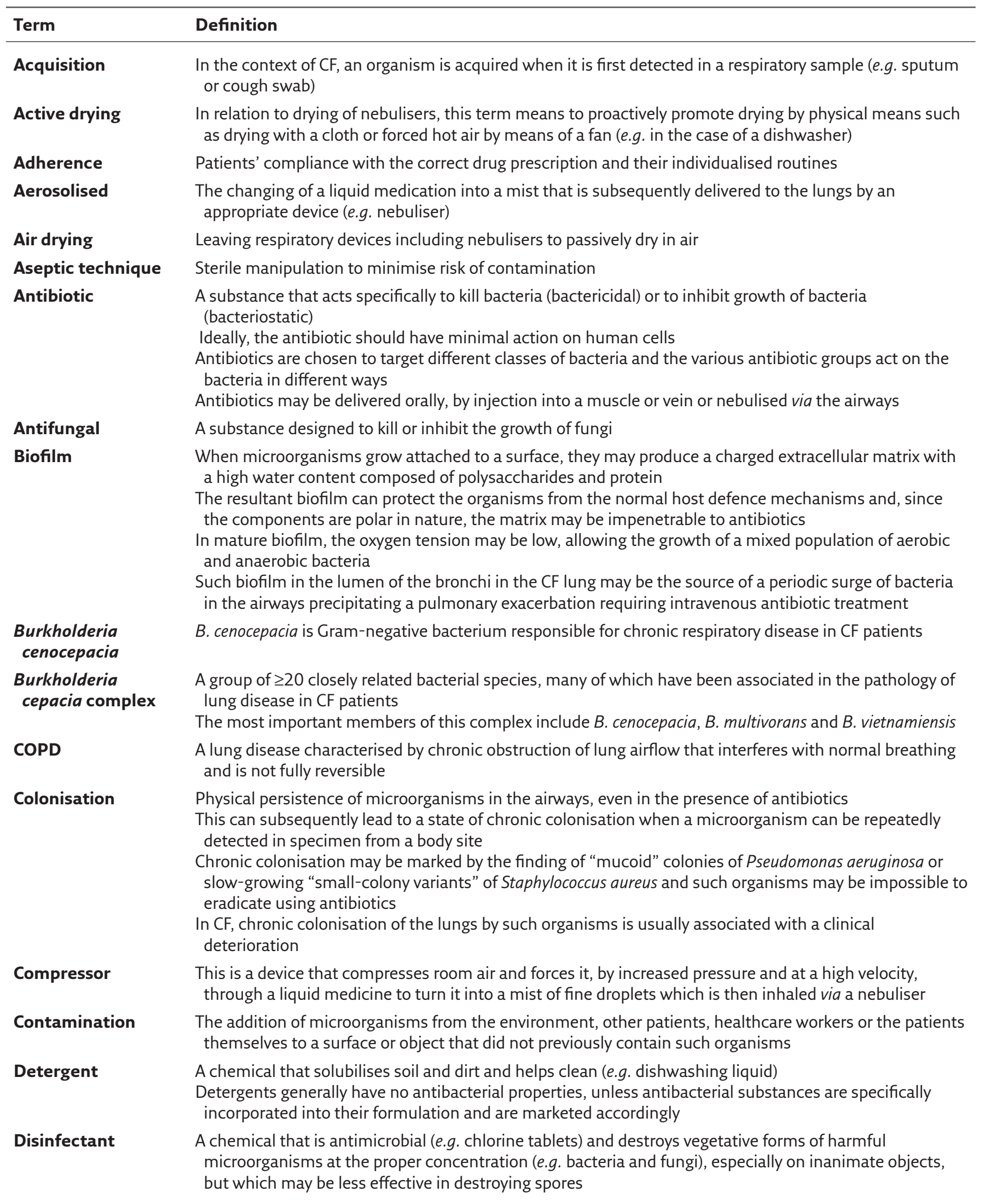


Table 1 Continued

\begin{tabular}{|c|c|}
\hline Term & Definition \\
\hline Disinfection & $\begin{array}{l}\text { The process (chemical, heat, ozone or ultraviolet light) by which a high percentage of the microorganisms } \\
\text { are removed or killed from an object or surface } \\
\text { This process will not inactivate bacterial/fungal spores and is less effective than sterilisation }\end{array}$ \\
\hline Distilled water & $\begin{array}{l}\text { Water that has been purified through boiling/evaporation and allowed to condense back into a liquid, } \\
\text { thus removing many impurities }\end{array}$ \\
\hline
\end{tabular}

\section{Eradication}

\section{Evidence-based practice (EBP)}

\section{Microbiome}

\section{Mucociliary clearance \\ Nebulised \\ Nebuliser}

Adaptive Aerosol Delivery

Disposable nebuliser

Jet nebuliser

Reusable nebuliser

Vibrating mesh nebuliser

\section{Nonpathogens}

\section{Nosocomial} infection

\section{Opportunistic} organisms

\section{Pathogens/ pathogenic organisms}

\section{Pseudomonas aeruginosa}

After the initial acquisition of certain bacteria, concerted attempts are made to eliminate the bacteria, usually by means of repeated courses of antibiotics

Early detection is the key to successful elimination and ideally, the treatment should be commenced as soon as possible after the organism is detected

The declaration that a patient has been cleared of an organism is always retrospective and requires a period (e.g. 1 year) during which only negative samples are obtained

The conscientious, explicit and judicious use of current best evidence in making decisions about the care of patients

EBP integrates best available external clinical evidence from systematic research

The sum of all the organisms in a region of the body

Using molecular methods including PCR and genetic sequencing, hundreds of groups may be identified

Studies of the microbiome in respiratory samples from CF patients show dynamic diversity of bacterial species

When chronic colonisation occurs, species diversity decreases with high concentrations of the relatively few organisms, such as $P$. aeruginosa or $B$. cepacia

The removal of particles from the airways as the result of the movement of mucus due to the beating of the underlying cilia

Administering a drug to the airways or lungs in the form of an aerosolised mist of fine droplets

A device that delivers aerosol droplets suitable for patient inhalation

Small, quick devices that give improved deposition of medications by delivering an aerosol on inhalation during the individual's breathing cycle (e.g. I-neb; Philips Respironics, Murrysville, PA, USA)

Discarded every $24 \mathrm{~h}$

Compressed air or oxygen used to aerosolise liquid medications

Can be used more than once

Moves liquid medication through a metal mesh to break up into a mist where each drop is a similar size, delivering a mist of medication constantly (e.g. E-flow; Pari, Byfleet, UK)

Organisms that do not cause disease, harm or death

A hospital-acquired infection

Organisms that take advantage of certain opportunities to cause disease (e.g. a weakened immune system)

Disease causing microorganisms, such as bacteria, fungi and viruses

A Gram-negative bacterium, commonly found in the environment, which is a significant pathogen in patients with CF 


\begin{tabular}{|c|c|}
\hline Term & Definition \\
\hline Potable water & Drinking water \\
\hline Pulmonary infection & Worsening clinical symptoms or signs, caused by pathogens identified in respiratory secretion samples \\
\hline $\begin{array}{l}\text { Stenotrophomonas } \\
\text { maltophilia }\end{array}$ & $\begin{array}{l}\text { A Gram-negative bacterium identified in people with CF } \\
\text { Although its clinical significance is a matter of debate, it is sometimes seen persistently in combination } \\
\text { with other more significant pathogens such as } P \text {. aeruginosa or S. aureus } \\
\text { In a few cases, this bacterium is the sole culminating organism in a CF patient with chronic pulmonary } \\
\text { infection }\end{array}$ \\
\hline Transmission & The process of passing microorganisms from one person or place to another \\
\hline
\end{tabular}

cultures obtained from swab samples from the device interface and chamber for nebuliser solution, with the most prevalent being Candida spp. cultured in $71.6 \%$ of nebulisers. Remarkably, only $4.8 \%$ (six out of 77 ) of nebulisers cultured nonmucoid $P$. aeruginosa, which seemed to correlate with the findings on frequency of nebuliser hygiene that was reported to be performed by $97.4 \%$ of patients, with cleaning, disinfection and drying being reported by the lesser percentage of $70.3 \%$ of patients [14].

Such contaminated nebulisers could potentially result in the deposition of infectious agents beyond the level of the ciliated epithelium, namely in regions of the lung airways that are distal to the terminal bronchioles and, as such, could potentially initiate a pulmonary infection.

Chronic colonisation of $P$. aeruginosa is a major contributor in the deterioration of lung function in $\mathrm{CF}$, and ultimately associated with the morbidity and mortality of the disease. Prevention of such chronic colonisation and infection is therefore a major goal, and decontaminating nebulisers, which may be a source of this organism, is one important step in such prevention. A study by PITCHFORD et al. [23] examined contamination of CF patients' home inhalation equipment, and detected $P$. aeruginosa in respiratory equipment, aerosol masks (20\%), nebulisers (17\%), medical syringes (10\%), connective tubing (6\%) and saline solution (4\%) from patients who were colonised with this organism. Although an early study, this highlights the important fact that the chain of transmission of $P$. aeruginosa should be broken, particularly when the first sputum cultures are positive, in order to have a realistic opportunity to eradicate initial acquisition [23].

\section{Experiences of nebuliser hygiene at home}

\section{At home}

There have been numerous reports of nebuliser hygienic practices in the home that have highlighted that each individual has their own ideas about how to clean and disinfect their nebuliser in terms of frequency of cleaning, washing, drying, disinfection and storage of nebulisers [7, 14, 15, 24]. One of the earliest studies concluded that respiratory therapists should immediately address and improve their nebuliser cleaning methods and replacement practices with more focus on teaching patients these practices [25].

\section{Inpatient stay}

Whilst there are various recommendations around nebuliser cleaning at home, primarily from manufacturers' instructions (table S2) and 2013 Infection Prevention and Control (IP\&C) Guideline for Cystic Fibrosis commissioned by the CF Foundation (CFF) [26], there is a lack of practical guidelines specific to inpatient nebuliser cleaning and disinfection. The IP\&C guidelines recommend the use of single-use disposable nebulisers during hospital admission that, after each use, should be rinsed with sterile water to remove residual volume, the mask/mouthpiece wiped with an alcohol pad and the nebuliser discarded every $24 \mathrm{~h}$ [26]. The advice of replacement of single-use nebulisers every $24 \mathrm{~h}$ has been challenged by WEBER et al. [22], due to the fact that his group observed infrequent contamination of single-patient use nebulisers 
Table 2 Organisms which have been recovered from the nebulisers of patients with CF [10-22]

\begin{tabular}{|c|c|}
\hline $\begin{array}{l}\text { Gram- } \\
\text { negative } \\
\text { bacteria }\end{array}$ & $\begin{array}{l}\text { Achromobacter xylosoxidans } \\
\text { Acinetobacter spp. (A. johnsonii, A. junii) } \\
\text { Aeromonas hydrophila } \\
\text { Burkholderia cepacia complex } \\
\text { Chryseobacterium indologenes } \\
\text { Comamonas spp. (C. acidovorans, C. testosteroni) } \\
\text { Delftia spp. } \\
\text { Enterobacter spp. } \\
\text { Escherichia coli } \\
\text { Flavobacterium spp. (F. indologenes, F. meningosepticum) } \\
\text { Haemophilus parahaemolyticus } \\
\text { Klebsiella spp. (K. ozaenae, K. pneumoniae) } \\
\text { Moraxella spp. (M. osloensis) } \\
\text { Neisseria subflava } \\
\text { Ochrobactrum anthropi } \\
\text { Oligella urethralis } \\
\text { Pantoea agglomerans } \\
\text { Proteus spp. } \\
\text { Pseudomonas spp. (P. aeruginosa, P. aureofaciens, P. fluorescens. } \\
\text { P. stuzeri, P. vesicularis) } \\
\text { Rhizobium (Agrobacterium) radiobacter } \\
\text { Serratia marcescens } \\
\text { Shewanella putrefaciens } \\
\text { Sphingobacterium spp. (Sphingomonas paucimobilis) } \\
\text { Stenotrophomonas maltophilia }\end{array}$ \\
\hline $\begin{array}{l}\text { Gram- } \\
\text { positive } \\
\text { bacteria }\end{array}$ & $\begin{array}{l}\text { Actinomyces viscosus } \\
\text { Bacillus spp. } \\
\text { Corynebacterium spp. } \\
\text { Enterococcus faecalis } \\
\text { Granulicatella adiacens } \\
\text { Microbacterium spp. (M. oxydans, M. paraoxydans) } \\
\text { Micrococcus spp. (M. Iuteus, M. lylae) } \\
\text { Rothia spp. (R. dentocariosa, R. mucilaginosa) } \\
\text { Staphylococcus spp. (S. aureus (MRSA, MSSA), S. epidermidis, } \\
\text { S. saprophyticus, S. pasteuri) } \\
\text { Streptococcus spp. (viridans group streptococci) }\end{array}$ \\
\hline $\begin{array}{l}\text { Other } \\
\text { bacteria }\end{array}$ & Paenibacillus spp. \\
\hline Yeasts & $\begin{array}{l}\text { Candida spp. (C. albicans, C. guilliermondii, C. holmii, C. krusei, } \\
\text { C. lipolytica, C. parapsilosis, C. sake, C. zeylanoides) } \\
\text { Cryptococcus spp. (C. albidus, C. carnescens, C. unigutatulus) }\end{array}$ \\
\hline $\begin{array}{l}\text { Filamentous } \\
\text { fungi }\end{array}$ & $\begin{array}{l}\text { Aspergillus spp. (A. fumigatus, A niger, A. versicolor) } \\
\text { Aureobasidium pullulans } \\
\text { Cladosporium spp. (Cladosporium sphaerospermum) } \\
\text { Exophiala spp. (E. jeanselmei, E. oligosperumum) } \\
\text { Lecanicillium spp. (L. lecanii) } \\
\text { Microsporum fulvum } \\
\text { Myxotrichum sp. } \\
\text { Penicillium spp. (P. commune, P. coryphilum, P. digitatum P. } \\
\text { glabrum, P. griseofulvin) } \\
\text { Rhizopus oryzae } \\
\text { Rhodotorula spp. (R. glutinis, R. minuta, R. mucilaginosa) } \\
\text { Scopulariopsis chartarum } \\
\text { Ulocladium chartarum } \\
\text { Sporobolomyces roseus } \\
\text { Trichosporon asahii }\end{array}$ \\
\hline
\end{tabular}

MRSA: methicillin-resistant S. aureus; MSSA: methicillin-sensitive S. aureus.
(0-11\%), with only low numbers of epidemiologically important pathogens ( $<100$ colony-forming units) after 24,48 or $72 \mathrm{~h}$ of use [22]. As such, the authors stated that they believed single-use nebulisers could be safely used for up to $72 \mathrm{~h}$ before disposal [22].

If reusable nebulisers are used (e.g. home equipment), the CFF-IP\&C guideline states after each use, the nebuliser should be cleaned, disinfected, rinsed with sterile water (post-cold water disinfection) and air dried away from the sink. The nebuliser can be reprocessed after each use if this is done according to manufacturer's instructions. This mirrors the recommendations of the Centers for Disease Control and Prevention for inpatient hand-held nebulisers, which are to clean, disinfect, rinse with sterile water (if appropriate) and dry between treatments on the same patient [27].

A recent survey carried out to examine current inpatient nebuliser hygiene practises in the UK [28] reported widespread variation between centres and highlighted everyday challenges. It is of interest to note that it is not common practise in the UK to use single-use disposable nebulisers as advocated by the CFF-IP\&C. Such practise is generally reserved for patients who either have forgotten their own nebuliser or do not wish to use their own nebuliser during an inpatient stay. The limited use of such disposable nebulisers may be in part a result of the cost implications. In this study [28], respondents reported nebulisers were washed after use or at least once daily in $90 \%$ of cases, but by a variety of methods and washing was carried out by the patient, the carer or a healthcare professional. Disinfection in hospital was performed less frequently, with 33\% of respondents reporting nebulisers were never disinfected during an inpatient stay due to lack of either appropriate methods for disinfection or access to decontamination facilities. Indeed, several patients (41\%) were required to take their nebuliser components home for disinfection purposes. Airdrying was the most commonly used method for drying the nebuliser but concern was expressed that due to logistical issues, such as small hospital rooms and lack of storage space, many nebulisers were being dried and stored beside splash zones. CF centres should have the appropriate facilities and systems in place to allow washing and disinfecting of nebulisers in a timely fashion to maximise therapy and patient safety.

\section{Evidence-based guidance}

\section{Washing procedures}

Hand washing

Hand hygiene is considered the most effective tool against hospital-acquired infection, including during decontamination of equipment. A recent study examining whether Staphylococcus aureus dry-surface biofilms may be transferred from contaminated surfaces to healthcare workers' hands showed that 
these microbes may be further transferred onto multiple surfaces, in sufficient quantities to cause infection, after only one touch of the contaminated item [29]. The CF Trust recommendations insist that good hand hygiene should be practised in all outpatient clinics and all inpatient facilities. Hand washing, disinfection with alcohol rubs or the use of disposable gloves are required before and after contact with each patient [30].

The CFF-IP\&C guideline [26] identifies before and after cleaning or disinfecting nebuliser equipment as an important opportunity for hand hygiene by both healthcare professionals and patients or carers, and suggests an alcohol-based hand gel as the preferred method in hospital. Water temperature has been investigated in a small number of studies with regards to hand washing. The updated Association of Perioperative Registered Nurses Recommended Practises for Hand Hygiene (2009) added a new statement to its recommendations that water temperature at the tap should be maintained between $105^{\circ} \mathrm{F}$ and $120^{\circ} \mathrm{F}\left(40.64-48.9^{\circ} \mathrm{C}\right)$ [31].

\section{Recommendation 1}

Healthcare professionals, persons with CF, or carers should wash hands and use hand disinfector gel before and after cleaning and disinfecting the nebuliser. Gloves should be worn by healthcare professionals with hand hygiene performed after removal of gloves.

\section{When to wash nebulisers}

A study by DE BOER et al. [32] showed that there is a marked difference in concentration of the aerosol cloud and time of nebulisation of tobramycin solution between an unwashed new Pari LC PLUS and one that was washed before use. The new unwashed medication chambers were noted to have large droplet formation on the inner walls, resulting in some of the tobramycin becoming adherent to the walls, and therefore lost to nebulisation.

The manufacturers of the most commonly used and recommended nebulisers in CF all advocate washing the nebulisers after each use (table S2). The CFF-IP\&C guidelines concur with this advice for both at-home and hospital inpatient use [26]. O'MALLEY [33] further clarifies that at home, cleaning should be performed as soon as possible after each use and that if it is not achievable directly after use, then at least to rinse the nebuliser parts well after use to prevent residual medication drying on the device

\section{Recommendation 2}

New nebuliser parts should be washed before first use.

\section{Recommendation 3}

During inpatient admission and at home, wash the nebuliser parts after each use.

\section{Recommendation 4}

Nebulisers should be washed and re-disinfected immediately prior to use after $>24$ h of inactivity.

\section{Where to wash nebulisers}

In the hospital

In a recent survey investigating inpatient nebuliser hygiene practices in the UK, it was noted that many patients used handwashing or bathroom sinks when washing their nebulisers [28]. This is a practice that should be avoided, particularly if the nebuliser parts are not subsequently disinfected, as the presence of clinically relevant microorganisms, such as $P$. aeruginosa and $B$. cenocepacia, in hospital sinks and drains has been well documented and in some cases, linked to outbreaks of infection [34, 35]. Possible sources of contamination on CF wards may be from the expectoration of sputum in the sink, the washing of contaminated hands or equipment, such as nebulisers, or the disposal of the water used for cleaning in the sink or toilet. Furthermore, a recent study identified a variety of environmental and clinical genotypes of $P$. aeruginosa in drains, taps and splash areas around sinks within multiple wards of a hospital, confirming hospital drains as an important source of this organism [36]. Nontuberculous mycobacteria (NTM) are important emerging pathogens in $\mathrm{CF}$ and have been reported in $61 \%$ of hospital water systems from 21 states within the USA [37]. Water stagnation and the limiting of hot water temperatures to prevent scalds may contribute to biofilm production and provide conditions suitable for mycobacterial growth [38].

\section{Recommendation 5 (inpatient)}

During inpatient stay, nebuliser parts should not be washed directly in sinks, especially bathroom sinks. This is particularly important if nebuliser parts are not immediately disinfected after each use. Additionally, nebulisers should not be washed in bathrooms containing a toilet, due to the risk of aerial contamination of the nebuliser by contaminated toilet droplets on flushing.

\section{Recommendation 6 (inpatient)}

During inpatient admission, nebuliser parts should be washed in disposable bowls or basins that may be sterilised by autoclaving. 


\section{Recommendation 7 (home and inpatient)}

Disinfection of nebuliser parts should be performed immediately after washing and these procedures should be performed in the patient's room.

In the home

The presence of $P$. aeruginosa in households of CF patients has been well documented, and it has been primarily isolated from drains of showers, drainpipes of hand basins in kitchens, bathrooms and drainpipes of toilets [39]. Recently, another study, which examined 11674 samples taken from a broad range of environmental sites in homes, showed that there was no difference in rates of $P$. aeruginosa recovery from environmental samples between houses with and without paediatric patients with CF [40]. The exact role of the environment in the acquisition of $P$. aeruginosa, however, remains unclear. A Belgian study compared the genotypes from newly infected patients with genotypes of isolates from the home environment. Results showed only nine (18\%) of the 50 patients in the study had $P$. aeruginosa genotypes identical to the genotype of the home environment. Environmental samples were obtained from the nebuliser, kitchen, bathroom and toilet, but the majority (72\%) of positive samples were recovered from the bathroom. Interestingly, $P$. aeruginosa was found in only one of the 75 samples taken from the 48 nebulisers of the 50 patients [41]. It must be noted, however, that it has been reported in patients with newly identified CF pathogens that the home environmental strains of Sphingomonas spp. and $P$. aeruginosa were identical to strains collected from the patients' sputum samples [42]. The existence of other CF pathogens in the home environment has identified not only $P$. aeruginosa but also Brevibacterium, Enterobacter, Mycobacterium, Neisseria, Staphylococcus and Streptococcus, with sink faucets as sites with the greatest burden of microorganisms [42].

In some guidelines, dishwashers have been recommended to disinfect nebuliser parts [26, 33]. This is not recommended, as studies have confirmed some human opportunistic pathogens are enriched and proliferate inside domestic dishwashers [43]. Exophiala dermatitidis (the "Black Yeast") is the third most frequently isolated fungus from CF patients, and it has been found on surfaces in kitchens with dishwashers and within dishwashers. Its presence in kitchens without dishwashers, however, is negligible [43]. With increased environmental concerns about energy efficiency and carbon footprint, there is an increasing tendency to operate dishwashers at lower temperatures. Unfortunately, there is no evidence regarding thermal destruction of this organism at dishwasher operating temperatures and, therefore, destruction of this organism cannot be guaranteed.

Given that there are effective alternative thermal disinfection methods available, coupled with the problems of deposition of particulate material on nebulisers undergoing dishwashing, employment of dishwashers is not suitable for either washing or disinfection of devices.

\section{Recommendation 8 (home)}

Do not wash nebuliser parts directly in kitchen or bathroom sinks.

\section{Recommendation 9 (home)}

Do not wash nebuliser parts in the dishwasher.

\section{Recommendation 10 (home)}

Nebuliser parts should be washed in a dedicated plastic, glass or metal bowl in the patient's kitchen. Such bowls should be washed and fully dried between each use.

\section{How to wash nebulisers}

Whilst all manufacturers advocate washing nebulisers after each use (table S2), there remain subtle, albeit important, differences in the methods advocated. For example, the water to be used may be described as hot, warm, clean water, tap water or distilled water and it may be recommended to wash, soak for varying time scales or agitate. Soap, detergent or dishwashing liquid may be cited for use. The following section attempts to offer recommendations for each of these differences.

In order to prepare nebulisers for washing, nebulisers should be disconnected from their compressor units and disassembled into their constituent components prior to washing and disinfection. This will allow for improved penetration of water-detergent mixtures to access remote regions of the nebulisers, thereby allowing for improved soil removal. Manufacturers discourage the use of brushes to aid in the washing of aerosol heads as this may cause damage [44].

\section{Recommendation 11}

Nebulisers should be disconnected from their compressor units and disassembled into their constituent components prior to washing/ disinfection. 


\section{Water}

It has been reported that nebulisers and masks are significantly more likely to be contaminated if they have been in use for $\geq 1$ month and have only been cleaned by rinsing with tap water after each use [23]. Of further concern is the fact that a study examining the occurrence of $P$. aeruginosa in water found $8 \%$ of tap water tested was positive for this organism, which highlights "the need to disinfect the CF patients' nebuliser after each use, to maintain an optimal Pseudomonas aeruginosafree device" [45]. Of note, additional evidence shows that contamination from tap water with $P$. aeruginosa and $B$. cenocepacia can be present weeks or years after the resolution of an outbreak $[34,46]$, most likely due to the formation of biofilms around the organisms that are present in the pipework.

The CFF-IP\&C guidelines regarding nebuliser hygiene both during hospitalisation and at home offers advice relating to sources of water, and their use at various stages of nebuliser cleaning and disinfection. The guidance states that tap water or well water that meets local public health standards, distilled water, or bottled water may be used for the cleaning nebulisers if followed by disinfection. Such water sources may be used for heat disinfection. However, they advocate the use of sterile water for use in the final rinse of nebuliser parts, particularly following disinfection [26].

An inpatient study demonstrated the presence of S. maltophilia in hospital taps, potable water and sink drains, highlighting that water may be a potential source of other clinically significant CF organisms [17]. End-line sterilising-grade water filters fitted to taps have been shown to be useful in helping to prevent the transmission of S. maltophilia to nebuliser chambers during washing [47], which may represent a simple, cost-effective practice for nebuliser washing.

There is a lack of scientific evidence available pertaining to the optimum temperature of water specifically for washing nebulisers; however, nebuliser manufacturers generally recommend warm or hot water in their instructions (table S2). A study looking at the cleaning of airway clearance devices showed complete or partial eradication of bacterial yield in $50 \%$ and $30 \%$, respectively, of contaminated airway clearance devices when they were soaked for $10 \mathrm{~min}$ in hot water (temperature unspecified) and detergent, and rinsed with hot water before air drying [13].

\section{Recommendation 12}

Tap water may be used for washing and rinsing nebulisers if it is followed by disinfection. Water should be warm or hot as available and comfortable for the user.

\section{Recommendation 13}

Sterile water must be used during a final rinse, when immediate disinfection is not possible. In such cases, this nebuliser should not be treated as disinfected.

\section{Detergent/soap}

Employment of detergent is important in order to help the removal of soil associated with the nebuliser. Such soil may include organic and inorganic contaminants, including sputum, saliva and crusted/dried-on nebulised medicines. Detergents act by reducing the surface tension of the liquid used in nebuliser cleaning, as well as promoting the emulsification of oil or grease and holding them in a suspension in water.

Certain protocols, particularly the manufacturers' instructions, cite the use of "soapy water", whilst others cite "dishwashing liquid" or "liquid detergent". Overall, there is a lack of standardisation of:

- terminology surrounding the use of detergent

- the chemistry of the detergent used

- detergent concentration

Certain device manufacturers have offered unsupported advice not to use certain detergent types, including those that are pearly and opaque or antibacterial liquid soaps [44], as these may damage the device. Soaps and detergents may themselves be a source of contamination of clinically significant organisms for CF patients [48, 49]. It has also been reported that hand soap dispensing units have been contaminated with Burkholderia cepacia, Pseudomonas putida, P. aeruginosa, Klebsiella pneumoniae, Enterobacter cloacae and Pseudomonas luteola [50].

\section{Recommendation 14}

Nebuliser components should be washed in a solution of warm tap water and dishwashing liquid, in accordance with the detergent manufacturer's guidance.

\section{Disposal of used water}

As discussed earlier, hospital hand washing sinks and bathroom sinks should not be used for washing nebulisers due to these environments being potential sources of pathogens. The disposal of the water used for cleaning the nebulisers may, in part, contribute to such contamination. Water that has been used in the washing of nebulisers may become contaminated with bacterial pathogens from contaminated nebuliser parts and therefore present a cross-infection risk, particularly within 
the hospital environment, requiring careful disposal of waste water. Additionally, it has been reported that patients who are infected with $P$. aeruginosa will excrete this organism in their stools, thereby contaminating the toilet environment [51], which is another potential source of cross infection.

\section{Recommendation 15 (inpatient)}

When disposable bowls are used for nebuliser washing, the bowl and waste water should be sent to the ward sluice for disposal. Disposable bowls should not be recycled. Nebuliser waste water should not be disposed of via sinks or toilets in order to prevent cross infection between occupants and subsequent occupants of rooms.

\section{Recommendation 16 (inpatient)}

When metal bowls are used for nebuliser washing, the metal bowl and waste water should be sent to the ward sluice. Waste water should be disposed of and the metal bowl should be sterilised by autoclaving.

\section{Recommendation 17 (home)}

Given that nebulisers will be washed in a separate container, thereby minimising risk to contaminating household sinks, and given that toilets are likely to be contaminated with the patient's own respiratory organisms, disposal of such waste water should be via the toilet, accompanied by avoidance of inhalation of aerosols by closing of the toilet lid prior to flushing.

\section{Disinfection procedures}

Historically, a plethora of methods of disinfection have been used for nebulisers, primarily chemical or thermal in nature; however, it is fundamentally important to ensure that the method used is effective in terms of decontamination and not detrimental to the nebuliser equipment or its output. Chemical and heat disinfection methods that have been used previously are reported in table 3. Generally, there are several limitations noted for the chemical disinfection methods, such as limited supporting evidence/preliminary findings, suboptimal methods of disinfection, corrosive activity, safe handling and storage of reagents, and half-life of reagents (table 3 ). Disinfection involving heat is the preferred method for optimal disinfection of nebulisers. Heat disinfection is a physical method and passes through all components of the previously washed/rinsed nebuliser. Employment of heat as a disinfection method is common with all nebuliser manufacturers' instructions, ranging from boiling to autoclaving. Steam disinfection with the aid of "baby bottle steam sterilisers" is an effective method for disinfection of nebuliser devices, as detailed (table 4). Whilst commercially termed a "steriliser" by the CF community and patients, this method is a disinfection method rather than a sterilisation method. This difference in terminology has important microbiological significance as to the anticipated outcome. One of the major advantages of steam disinfection using a baby bottle disinfector unit is that the process is a batch process with terminal (end-stage) disinfection. The sequence of disinfection as the terminal process will allow for the destruction of pathogens detailed in table 4 , which may enter during prior stages (tap water, residual sputum, detergent, handling, etc.). Whilst steam disinfection is effective microbiologically in the presence of soil on the nebuliser, it is still important to thoroughly wash nebulisers prior to disinfection, to remove residue that could potentially interfere with the optimal performance of the device.

\section{Recommendation 18 (home and inpatient)}

Washed and rinsed nebulisers should be disinfected after each use by employment of heat using an electric baby bottle steam disinfector, in accordance with the manufacturer's instructions.

\section{Recommendation 19 (inpatient)}

Reusable nebulisers may be autoclaved where the nebuliser manufacturer states that the device can be safely autoclaved.

\section{Drying}

Desiccation (drying) is a technique that can be used to effectively kill bacteria [70]. In theory, air drying the nebuliser should significantly reduce the risk of nosocomial infection. In the study by MANOR et al. [13], simple cleaning and air drying without disinfection was found to be effective in most cases. Air drying has been advocated as a method of drying to cause least recontamination of cleaned parts [62] and is now the drying technique of choice advocated by many nebuliser manufacturers.

A recent study by Moore et al. [71] examined the effect of drying on $P$. aeruginosa and showed that there were marked differences in time to complete dryness between disassembled and fully assembled nebulisers. Vigorous repeated shaking was unable to drive off all residual water on assembled nebulisers. $P$. aeruginosa counts did not decrease significantly in either tap water or in tap water plus detergent after $24 \mathrm{~h}$ storage at ambient temperature. In contrast, all Pseudomonas organisms were killed when dried for $24 \mathrm{~h}$, even when contaminated with $1 \%$ and $10 \%$ sputum. Dishwashing detergent did not 
Table 3 A list of documented methods used to disinfect nebulisers

\begin{tabular}{l} 
Method \\
\hline Chemical methods \\
Acetic acid/vinegar \\
Benzyl ammonium chloride (Control \\
III; Maril Inc., Tustin, CA, USA) \\
Ethyl alcohol/ethanol (70\%)
\end{tabular}

\section{Evidence}

Isopropyl alcohol/propanol (70\%)

Hydrogen peroxide (3\%)

Sodium hypochlorite

Ozone

Squalamine

\section{Heat methods}

Boiling (continuous boil in water for 5 mins)

Steam (electric baby bottle steam disinfectors)

Steam (microwave) (place in microwave-safe receptacle submerged in water and microwave for 5 mins, $2.45 \mathrm{GHz}$ )

Dishwasher disinfection $\left(\geq 70^{\circ} \mathrm{C} / 158^{\circ} \mathrm{F}\right.$ for $\left.30 \mathrm{~min}\right)$

Autoclave (a maximum of $277^{\circ} \mathrm{F}$ or $\left.136^{\circ} \mathrm{C}\right)$

MRSA: methicillin-resistant S. aureus.

Not recommended due to narrow spectrum of activity and slow action [26]

Has been reported to reduce fungal and bacterial contamination of nebulisers but not completely [53]

Will lose activity over time; therefore, needs to be regularly replaced

Saliva and sputa may protect microorganisms from the action of alcohol; therefore, prior washing of devices is required

May be ineffective against mycobacteria [54]

It has been reported that isopropyl alcohol disinfection does not affect nebuliser output [55]

Although listed in the CFF-IP\&C guidelines (3\% hydrogen peroxide for $30 \mathrm{~min}$ ), there is limited evidence supporting disinfection of nebulisers with hydrogen peroxide [26]

Although hypochlorite disinfection has been shown to decrease the contamination of nebulisers due to commensal and environmental organisms, a similar reduction has not been observed in the case of CF pathogens [10,56]

Corrosive chemicals such as hypochlorite are prohibited for disinfecting the mesh membrane of the e-Flow rapid nebuliser [5]

Commercially available SoClean (Peterborough, NH, USA) ozone machine has been examined with type strains of B. cepacia, P. aeruginosa, S. aureus and S. maltophilia Preliminary findings have shown a 5 -min infusion followed by a 120 -min dwell time to be effective against these organisms when spiked onto a Pari LCPlus nebuliser, without affecting nebuliser output

Further investigation is required in relation to nontuberculous mycobacteria and clinical strains of CF pathogens [57]

Squalamine (a steroid-polyamine conjugate compound with broad spectrum antimicrobial activity) targets membrane integrity, and has potential to reduce levels of S. aureus, P. aeruginosa, Candida albicans and Aspergillus niger on contaminated nebulisers [58]

Further work needs to be completed prior to advocating the employment of this molecule

The CFF-IP\&C guidelines and manufacturers' guidelines recommend that nebulisers should be disinfected by placing them in boiling water for 5 mins [26]

Decrease in nebuliser output with Pari LC Plus $®$ device following repeated disinfection by boiling [55]; however, experimental limitations must be considered [59]

See table 4

Evidence in CFF-IP\&C guideline taken from [65-67]; none of these studies employed nebulisers

This method cannot be used with nebulisers that contain metal components (e.g. mesh in the eFlow rapid)

Dutch study demonstrated that nebulisers contaminated with Enterococcus faecalis, $S$. aureus and $P$. aeruginosa were free of microorganisms after microwave treatment (650 W, 9 min) [68]

Although listed in the CFF-IP\&C guidelines [26], there is limited evidence supporting disinfection of nebulisers using high-temperature dishwashers

Certain nebuliser parts may be sterilised using autoclave methods (e.g. Pari Reusable Nebuliser parts, except mask and tubing) [69] 
Table 4 Advantages and disadvantages of steam disinfection for nebuliser devices

Advantages
Effective killing of a wide range of bacter
B. cepacia complex
B. cepacia
Burkholderia multivorans
Candida albicans
Enterococcus faecium
Haemophilus influenzae
Mycobacterium abscessus abscessus
Mycobacterium abscessus bolletii
Mycobacterium abscessus massiliense
Mycobacterium abscessus/chelonae
Mycobacterium avium intracellulare
Mycobacterium chelonae
P. aeruginosa (mucoid, nonmucoid)
S. aureus (MRSA, MSSA)
Steno. maltophilia

The commercial Steam Sterilizer Plus Model (Wabi Baby, Frisco, TX, USA) has been examined for its effectiveness in disinfecting nebulisers spiked with CF organisms [63]:

Achromobacter xylosoxidans
B. cepacia
P. aeruginosa
MSSA

$P$. aeruginosa (mucoid, nonmucoid) and nontuberculous mycobacteria were not detected in either wet or dry fully assembled nebulisers following steam disinfection even in the presence of sputa [62]

Different brands of steam disinfectors performed effectively [62]

Terminal (end-stage) disinfection will allow for destruction of above pathogens, which may enter during prior stages (tap water, residual sputum, detergent, handling, etc.)

Relatively cheap (approximately GBP 50, EUR 80, USD 70)

Widely available in electrical shops and from online sellers

Simple to operate

No consumable items required, except tap water

Fast cycle speeds (approximately 8-15 min to disinfection)

Disinfected nebuliser can be stored in disinfector units post-cycle until required $(\leq 24 \mathrm{~h}$ ), thus avoiding post-disinfection contamination from surfaces, hands, environment, tap water

Portable (maybe taken on holiday/travel if voltage compatible)

Can be used with metal components (e.g. metal vibrating mesh)

No membrane performance effect with Pari e-Flow rapid device [64]

\section{Disadvantages}

Apart from the Wabi Baby disinfector, all other baby bottle disinfector manufacturers have not endorsed their product for nebuliser disinfection None of the steam disinfector units have indicators to show/confirm that unit has successfully completed its full cycle
Wabi Baby device only available in USA
Decrease in nebuliser output with Pari LC Plus device following repeated steam disinfection [55]; however, experimental limitations must be considered [59]

MRSA: methicillin-resistant S. aureus; MSSA: methicillin-sensitive S. aureus.

demonstrate any antibacterial activity. The study concluded that drying of the nebuliser, if applied properly, can reduce counts of $P$. aeruginosa to undetectable levels and therefore minimise risk of acquisition of $P$. aeruginosa in the patient from the nebuliser device. Equally, this study showed that if the device is not dried thoroughly and moisture remains, then the device is able to support the survival of $P$. aeruginosa at high numbers, constituting an infection risk to the CF patient. These data may help educate and inform the CF patient about the importance of proper nebuliser drying for Pseudomonas control, in order to improve patient awareness and safety [71]. More recently, 
there have been two reports demonstrating that members of the NTM, Mycobacterium abscessus complex and S. aureus (methicillin-sensitive and -resistant) can survive for at least $24 \mathrm{~h}$ on plastic surfaces during air drying [72, 73]. Therefore, whilst drying may be advantageous for nebuliser performance, it should not be regarded as an absolute critical control for the elimination of NTM or S. aureus organisms on nebuliser devices and, hence, devices should be disinfected with heat in accordance with recommendations 18 and 19 .

As highlighted in previous sections, care must be taken to ensure that parts that are left out to air dry are kept away from sinks and potential splash areas [36], which in itself may pose a logistical problem in a small hospital room with limited surfaces. Enough time must be given to allow the parts to dry fully, which may be challenging during an inpatient admission when nebulised therapies and airway clearance are being optimised. In addition, some components are difficult to dry and the degree of dryness can be difficult to assess.

The use of disposable paper towels for active drying was shown as the next best alternative, with a low count of bacteria detected [62]. However, use of paper towels may cause decreased performance of some nebulisers by clogging of pores (such as the Eflow aerosol head) and as such, is not recommended by certain manufacturers.

The study by HoHenWARTER et al. [62] has challenged whether drying is necessary after steam disinfection, as they reported that nebulisers that were disinfected and left undisturbed in a baby bottle disinfector remained bacteria free until the next use within $24 \mathrm{~h}$. They suggest that this may be a better solution as they have shown that active drying may cause recontamination. In addition, the authors suggest that this would be a simple, effective and time-efficient method for patients and carers to adopt.

\section{Storage}

The storage of disinfected nebuliser parts in a sealed disinfector unit after the cycle has finished [62] removes the risk of re-contaminating an already disinfected device, as well as avoiding the need for additional storage space.

It is vital that even though a disinfection method may be presumed to be effective, that instruction for storage of disinfected nebulisers is also given, as storage of damp nebulisers is a potential source of contamination [9]. A recent study [74] examined the microbiology of plastic disposal boxes that were used to store washed nebulisers and found that $80 \%$ of patients had at least one of their storage boxes positive for bacteria. Overall, $83 \%$ (20 out of 24) of boxes were positive for bacteria; however, the majority of these $(65 \%)$ had a contamination rate of $<10^{3}$ colony forming units (cfu) per box. $15 \%$ of positive boxes were contaminated with $10^{3}-10^{4} \mathrm{cfu}$ per box, with the remainder (20\%) contaminated with between $10^{4}$ and $10^{5} \mathrm{cfu}$ per box. The most highly contaminated box harboured $5.4 \times 10^{4} \mathrm{cfu}$. Bacterial diversity demonstrated a predominately Gram-positive flora, representing 15 genera and 22 species. Micrococccus luteus and Dermacoccus nishinomiyaensis were the most commonly isolated species, with coagulase-negative staphylococci and the viridans group (oral) streptococci having the greatest species diversity within their respective genera. Gram-negative bacteria were in the minority, representing $8.3 \%$ of bacterial species isolated, namely S. maltophilia and Neisseria flava/perflava/subflava. Fungi were isolated from four (26.7\%) out of 15 boxes and included Penicillium sp., Penicillium expansum, Cladosporium sp. and Candida albicans.

\section{Recommendation 20: (home and inpatient)}

Following disinfection in a baby bottle steam disinfector, nebuliser parts should be left undisturbed in the disinfector until the next use (within 24 h). Should the parts be disturbed (i.e. the lid lifted off for any reason), then the disinfection process should be repeated and the parts left undisturbed until the next use (within $24 \mathrm{~h}$ ).

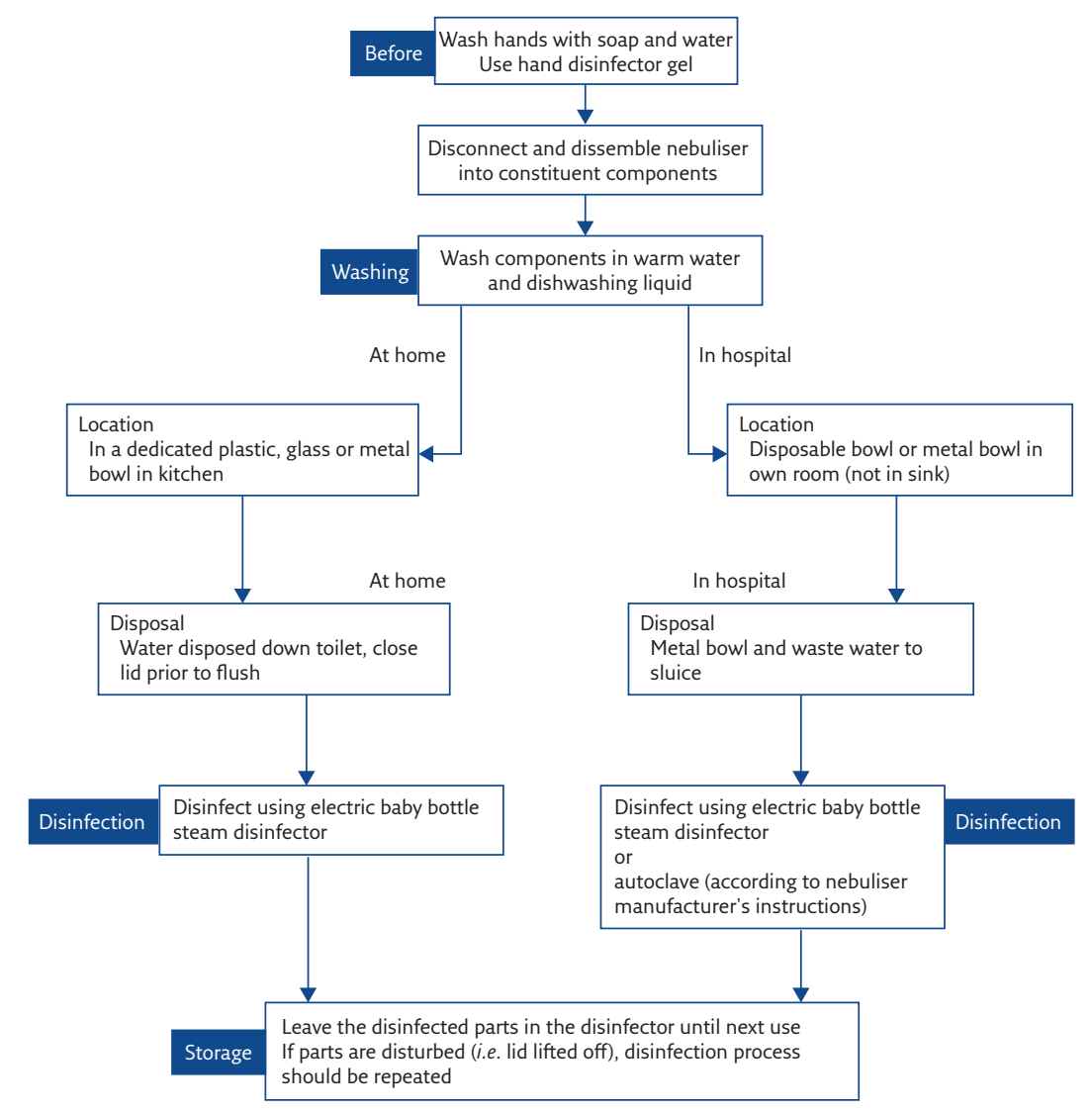

Figure 1 Summary of recommendations as a flow diagram. 
Table 5 Summary of evidence-based best practice guidelines relating to nebuliser hygiene in CF

New nebuliser parts should be washed and disinfected before first use (recommendation 2)

During hospital admission and at home, wash the nebuliser parts after each use (recommendation 3)

Nebulisers should be washed and re-disinfected immediately prior to use after greater than $24 \mathrm{~h}$ of inactivity (recommendation 4)

\section{At-home recommendations \\ Inpatient recommendations}

Wash hands thoroughly with soap and water and use hand disinfector gel before starting the cleaning process (Recommendation 1)

Disconnect nebuliser from the compressor unit and dissemble into constituent components (recommendation 11)

Washing

Wash nebuliser components in a solution of warm tap water (recommendation 12) and dishwashing liquid in accordance with the detergent manufacturer's guidance (recommendation 14)

Location

Do not wash nebuliser parts directly in kitchen or bathroom sinks (recommendation 8) or the dishwasher (recommendation 9)

This should be done in a dedicated plastic, glass or metal bowl in the patients' kitchen (recommendation 10)

Rinsing

Sterile water must be used during a final rinse when immediate disinfection is not possible (recommendation 13)

Disposal of waste water

Disposal of waste water should be via the toilet, ensuring the toilet lid is closed prior to flushing (recommendation 17)

\section{Disinfection}

Washed and rinsed nebulisers should be disinfected after each use (recommendation 7) using an electric baby bottle steam disinfector, (recommendation 18)

\section{Storage}

Leave the disinfected nebuliser parts undisturbed in the disinfector until the next use (within $24 \mathrm{~h}$ )

Should the parts be disturbed (i.e. lid lifted off for any reason) then the disinfection process should be repeated and the parts left undisturbed until the next use (within $24 \mathrm{~h}$ ) (recommendation 20)

After disinfection

Wash hands thoroughly with soap and water, and use hand disinfector gel after the cleaning and disinfection process prior to reassembling the nebuliser device (recommendation 1)
Wash hands thoroughly with soap and water and use hand disinfector gel before starting the cleaning process.

Gloves should be worn by healthcare professionals with hand hygiene performed after removal of gloves (recommendation 1)

Disconnect nebuliser from the compressor unit and dissemble into constituent components (recommendation 11)

Washing

Wash nebuliser components in a solution of warm tap water (recommendation 12) and dishwashing liquid in accordance with the detergent manufacturer's guidance (recommendation 14)

Location

This should be done in a disposable bowl, metal bowl or basin (not directly in a sink; recommendation 5) in the patients' room (recommendation 6)

Rinsing

Sterile water must be used during a final rinse, when immediate disinfection is not possible (recommendation 13).

Disposal of waste water

Metal bowl and waste water should be sent to the ward sluice (recommendation 15).

Waste water should be disposed of here and the metal bowl should be sterilised by autoclaving (Recommendation 16).

Disinfection

Washed and rinsed nebulisers should be disinfected immediately after each use (recommendation 7) using an electric baby bottle steam disinfector (recommendation 18), or

reusable nebulisers may be autoclaved, where the nebuliser manufacturer states that the device can be safely autoclaved (recommendation 19)

Storage

Leave the disinfected nebuliser parts undisturbed in the disinfector until the next use (within $24 \mathrm{~h}$ )

Should the parts be disturbed (i.e. lid lifted off for any reason) then the disinfection process should be repeated and the parts left undisturbed until the next use (within $24 \mathrm{~h}$ ) (recommendation 20)

After disinfection

Wash hands thoroughly with soap and water, and use hand disinfector gel after the cleaning and disinfection process prior to reassembling the nebuliser device (recommendation 1) 
Wash hands thoroughly with soap and water, and use hand disinfector gel after the cleaning and disinfection processes, prior to reassembling the nebuliser device (recommendation 1).

\section{Barriers and motivators related to nebuliser hygiene}

The absence of universal guidance relating to nebuliser care and hygiene appear to have contributed to the current situation where CF patients are bereft of vital knowledge on the importance of such practices. These practices should be an essential component of their routine CF treatment regime. The customary informationgiving process alone may not be enough to assist CF patients and families in implementing and maintaining long-term behavioural change. In the dissemination of universal guidance, it is crucial that the education of staff, patients and families is a priority. The guidance should be clear, consistent, systematic and predicated on an understanding of the complexity of the CF treatment regimen and, in particular, the heavy time burden on patients.

It is important that there is a process of ongoing monitoring of the implementation as well as routine assessment of the barriers and motivators to implementation as experienced by staff, patients and families. Collaborative adherence promotion interventions developed by CF healthcare professionals have the potential to advance clinical practice [75]. The focus should be on developing ways to overcome the barriers and use motivators to ensure that nebuliser care and hygiene becomes an established part of the daily treatment routine of the CF patient. The clinical outcomes of the new nebuliser care procedures need to be fed back to staff, patients and families to elucidate the impact of adherent behaviour on the health of CF patients.

We can gain inspiration from LuBy et al. [76], who studied the effect of hand washing on child health in Karachi, Pakistan. They made soap use more systematic in people living in the slums of Karachi. The soap distribution led to behaviour change as fieldworkers provided people with soap and specific instruction on hand washing technique and hygiene practices. The decreased incidence of diarrhoea, impetigo and pneumonia demonstrates the effectiveness of this intervention. In a 5-year follow up, there were sustained improvements in handwashing indicators [77]. They attributed the sustained behavioural change to the intensive nature of the intervention and the habituation of handwashing behaviour until it acquired a degree of unconscious automaticity.
Similarly, a study carried out by a group in Brazil successfully showed that an educational programme had a significant positive outcome on nebuliser contamination [21].

The inpatient setting provides an opportunity for re-education of appropriate cleaning and disinfecting standards to enable patient ownership of their healthcare. It is therefore imperative for healthcare professionals responsible for endorsing cleaning and disinfecting methods, to be able to follow the gold-standard care themselves. Lack of clarity and differing advice may discourage the CF patient and/or carer from engaging with the importance of nebuliser care.

\section{Conclusions}

Nebuliser therapy constitutes one small part of the daily treatment burden for people living with $\mathrm{CF}$ and therefore, the maintenance and hygiene of the nebuliser itself can often be overlooked as an essential aspect of this daily care. As well as promoting the importance of taking prescribed nebuliser treatments, emphasis should equally be placed on the importance of nebuliser care to patients' outcomes, as being pivotal in preventing reinfection, optimising delivery of treatment safely and efficiently, thus ensuring maximum benefit and reduced risk in using nebulisers. Confusion as a result of differing manufacturers' advice, lack of clear guidelines and conflicting anecdotal advice from caregivers may have a negative impact on adherence to nebuliser care.

This review has presented the current evidence base to rationalise the need for nebuliser hygiene and to present evidence-based recommendations (figure 1 and table 5), which can be used to help guide local nebuliser infection control policy.

Appropriate care and maintenance of the nebuliser must form an integral part of the education and behavioural change programme when commencing nebuliser therapy and this must be re-asserted alongside the reassessment of nebuliser inhalation technique and usage.

The Northern Ireland Working Group on Nebuliser Care and Hygiene in Cystic Fibrosis has produced two YouTube videos to accompany this article.

- Nebuliser cleaning \& hygiene in cystic fibrosis: https://youtu.be/-b4hXUuRiFo

- Cleaning the nebuliser (for children): https://youtu.be/Pw_w9ZXrHsw 


\section{Key points}

- Nebuliser hygiene is essential to ensure optimal performance of the device as well as being a vital step to avoid microbial contamination of the components and potential infection/re-infection to the user.

- Current nebuliser hygiene practice is varied and challenging, both at home and during inpatient stays.

- Consideration must be given to the location, timing and procedure for washing, disinfecting and drying nebuliser components.

- Education of patients, families and staff is essential to drive long term adherence to nebuliser hygiene guidelines.

Affiliations

Jane Bell' ${ }^{1}$, Lauren Alexander ${ }^{1}$, Jane Carson ${ }^{1}$, Amanda Crossan², John McCaughan ${ }^{3}$, Hazel Mills', Damian O'Neill'2, John E. Moore 2,4 and B. Cherie Millar2,4, on behalf of the Northern Ireland Working Group on Nebuliser Care and Hygiene in Cystic Fibrosis

${ }^{1}$ Northern Ireland Paediatric Cystic Fibrosis Centre, Dept of Physiotherapy, Royal Belfast Hospital for Sick Children, Belfast, UK. ${ }^{2}$ Northern Ireland Regional Adult Cystic Fibrosis Centre, Belfast City Hospital, Belfast, UK. ${ }^{3}$ Dept of Medical Microbiology, Royal Victoria Hospital, Belfast, UK. ${ }^{4}$ Northern Ireland Public Health Laboratory, Dept of Bacteriology, Belfast City Hospital, Belfast, UK.

\section{Acknowledgements}

This review was guided by the Northern Ireland Working Group on Nebuliser Care and Hygiene in Cystic Fibrosis. The authors were members of the working group and were involved in meeting and planning of the review, as well as examination of the evidence-based literature. All authors contributed to writing the manuscript. The other members of the working group were involved in meeting and planning of the review: Niju Baby, Catherine Bell, Kevin Brookfield, Lesley Carson, Colin Goldsmith, Julie Hanna, Katherine Lavery, Lisa Murphy, Mary Macfarlane, Valerie Mills, Liz Quinn. All members of the working group reviewed, edited and approved the final version of the manuscript. The views, opinions, findings, conclusions and recommendations set forth in this manuscript are solely those of the authors and do not necessarily reflect the views, policy or position of the affiliations of the authors.

This article has supplementary material available from breathe.ersjournals.com.

\section{Support statement}

The Northern Ireland Working Group on Nebuliser Care and Hygiene in Cystic Fibrosis is supported by an unrestricted medical educational grant from Vertex Pharmaceuticals Inc., Boston, MA, USA.

\section{Conflict of interest}

J. Bell has nothing to disclose. L. Alexander has nothing to disclose. J. Carson has nothing to disclose. A. Crossan reports speaker honoraria from TEVA and AstraZeneca, and sponsorship to attend a European cystic fibrosis conference from Chiesi, all outside the submitted work. J. McCaughan has nothing to disclose. H. Mills has nothing to disclose. D. O'Neill has nothing to disclose. J.E. Moore has nothing to disclose. B.C. Millar has nothing to disclose.

References

1. Agent $P$, Parrott $H$. Inhaled therapy in cystic fibrosis: agents, devices and regimens. Breathe 2015; 11: 110-118.

2. Heijerman $H$, Westerman $E$, Conway S, et al. Inhaled medication and inhalation devices for lung disease in patients with cystic fibrosis: A European consensus. J Cyst Fibros 2009; 8: 295-315.
3. Boe J, Dennis J, O’Driscoll B, et al. European Respiratory Society guidelines on the use of nebulizers. Eur Respir J 2001; 18: 228-242.

4. Pasteur M, Bilton D, Hill A. British Thoracic Society guideline for non-CF bronchiectasis. Thorax 2010; 65: i1-i58. 
5. Baravalle-Einaudi M, Dufeu N, Dupont C, et al. Vibrating-mesh nebulizer maintenance by CF patients: results from a French survey. Pulm Pharmacol Ther 2017; 44: 57-60.

6. Rottier BL, van Erp CJ, Sluyter TS, et al. Changes in performance of the Pari eFlow rapid and Pari LC Plus during 6 months use by CF patients. J Aerosol Med Pulm Drug Deliv 2009; 22: 263-269.

7. Reychler G, Bosschaerts M, Chevaillier J, et al. Inhalation equipment hygiene: a Belgian national survey. J Aerosol Med Pulm Drug Deliv 2009; 22: 239-243.

8. Le Pennec D, Baravalle-Einaudi M, Dupont C, et al. Cystic fibrosis in the modern therapeutic era: Give the shower a thought! J Cyst Fibros 2019; 18: e56-e57.

9. Cobben NA, Drent M, Jonkers M, et al. Outbreak of severe Pseudomonas aeruginosa respiratory infections due to contaminated nebulizers. J Hosp Infect 1996; 33: 63-70.

10. Hutchinson GR, Parker S, Pryor JA, et al. Home-use nebulizers: a potential primary source of Burkholderia cepacia and other colistin-resistant, gram-negative bacteria in patients with cystic fibrosis. J Clin Microbiol 1996; 34: 584-587.

11. Blau H, Mussaffi $H$, Mei Zahav M, et al. Microbial contamination of nebulizers in the home treatment of cystic fibrosis. Child Care Health Dev 2007; 33: 491-495.

12. Peckham D, Williams K, Wynne S, et al. Fungal contamination of nebuliser devices used by people with cystic fibrosis. J Cyst Fibros 2016; 15: 74-77.

13. Manor E, Gur M, Geffen Y, et al. Cleaning and infection control of airway clearance devices used by CF patients. Chron Respir Dis 2017; 14: 370-376.

14. Riquena B, Monte LFV, Lopes AJ, et al. Microbiological contamination of nebulizers used by cystic fibrosis patients: an underestimated problem. J Bras Pneumol 2019; 45: e20170351.

15. Murray TS, O'Rourke TK Jr, Feinn R, et al. Nebulizer cleaning and disinfection practices in families with cystic fibrosis: The relationship between attitudes, practice and microbe colonization. J Cyst Fibros 2019; 18: 823-828.

16. O'Malley CA, VandenBranden SL, Zheng XT, et al. A day in the life of a nebulizer: surveillance for bacterial growth in nebulizer equipment of children with cystic fibrosis in the hospital setting. Respir Care 2007; 52: 258-262.

17. Denton M, Rajgopal A, Mooney L, et al. Stenotrophomonas maltophilia contamination of nebulizers used to deliver aerosolized therapy to inpatients with cystic fibrosis. J Hosp Infect 2003; 55: 180-183.

18. Jakobsson BM, Onnered AB, Hjelte L, et al. Low bacterial contamination of nebulizers in home treatment of cystic fibrosis patients. J Hosp Infect 1997; 36: 201-207.

19. Vassal S, Taamma R, Marty N, et al. Microbiologic contamination study of nebulizers after aerosol therapy in patients with cystic fibrosis. Am J Infect Control 2000; 28: 347-351.

20. Brzezinski LX, Riedi CA, Kussek P, et al. Nebulizers in cystic fibrosis: a source of bacterial contamination in cystic fibrosis patients? J Bras Pneumol 2011; 37: 341-347.

21. Della Zuana A, Garcia Dde O, Juliani RC, et al. Effect that an educational program for cystic fibrosis patients and caregivers has on the contamination of home nebulizers. J Bras Pneumol 2014; 40: 119-127.

22. Weber DJ, Gergen MF, Sickbert-Bennett EE, et al. Frequency of contamination of single-patient-use nebulizers over time. Infect Control Hosp Epidemiol 2014; 35: 1543-1546

23. Pitchford KC, Corey M, Highsmith AK, et al. Pseudomonas species contamination of cystic fibrosis patients' home inhalation equipment. Pediatr 1987; 111: 212-216.

24. MacFarlane M, Carson L, Crossan A, et al. Nebuliser cleaning and disinfection practice in the home among patients with cystic fibrosis. J Infec Prev 2020; 21: 14-22.

25. O'Malley CA. Infection control in cystic fibrosis: cohorting, cross-contamination and the respiratory therapist. Respir Care 2009; 54: 641-655.

26. Saiman L, Siegel JD, LiPuma JJ, et al. Infection prevention and control guideline for cystic fibrosis: 2013 update. Infect Control Hosp Epidemiol 2014; 35: Suppl. 1, 1-67

27. Tablan OC, Anderson LJ, Besser R, et al. Guidelines for preventing health-care-associated pneumonia, 2003: recommendations of CDC and the Healthcare Infection Control Practices Advisory Committee. MMWR Recomm Rep 2004; 53: 1-36.

28. Bell J, Moore JE, Millar BC. Cleaning of inpatient nebulizer devices in cystic fibrosis patients: the urgent need for universal guidelines. J Hosp Infect 2018; 100: e64-e66

29. Chowdhury D, Tahir S, Legge M, et al. Transfer of dry surface biofilm in the healthcare environment: the role of healthcare workers' hands as vehicles. J Hosp Infect 2018; 100: e85-e90.

30. Cystic Fibrosis Trust. Consensus documents. www.cysticfibrosis. org.uk/the-work-we-do/resources-for-cf-professionals/ consensus-documents.

31. Patrick M, van Wicklin SA. Implementing AORN recommended practices for hand hygiene. AORN 2012; 95: 492-504

32. de Boer $\mathrm{AH}$, Hagedoorn P, Frijlink HW. The choice of a compressor for the aerosolization of tobramycin (TOBI@) with the PARI LC PLUS® reusable nebuliser. I Pharm 2003; 268: 59-69.

33. O'Malley CA. Device cleaning and infection control in aerosol therapy. Respir Care 2015; 60: 917-931.

34. Bédard $E$, Laferrière $C$, Charron D, et al. Post-outbreak investigation of Pseudomonas aeruginosa faucet contamination by quantitative polymerase chain reaction and environmental factors affecting positivity. Infect Control Hosp Epidemiol 2015; 36: 1337-1343

35. Lucero CA, Cohen AL, Trevino I, et al. Outbreak of Burkholderia cepacia complex among ventilated patients linked to hospital sinks. Am J Infect Control 2011; 39: 775-778.

36. Lalancette $C$, Charron $D$, Laferrière $C$, et al. Hospital drains as reservoirs of $P$ seudomonas aeruginosa: Multiple-locus variablenumber of tandem repeats analysis genotypes recovered from faucets, sink surfaces and patients. Pathogens 2017; 6: E36.

37. Covert TC, Rodgers MR, Reyes AL, et al. Occurrence of nontuberculous mycobacteria in environmental samples. Appl Environ Microbiol 1999; 65: 2492-2496.

38. Wallace RJ Jr, Brown BA, Griffith DE. Nosocomial outbreaks/ pseudo-outbreaks caused by nontuberculous mycobacteria. Annu Rev Microbiol 1998; 52: 453-490.

39. Regnath T, Kreutzberger M, Illing $S$, et al. Prevalence of Pseudomonas aeruginosa in households of patients with cystic fibrosis. Int J Hyg Environ Health 2004; 207: 585-588.

40. Purdy-Gibson ME, France M, Hundley TC, et al. Pseudomonas aeruginosa in CF and non-CF homes is found predominantly in drains. J Cyst Fibros 2015; 14: 341-346.

41. Schelstraete P, Van Daele S, De Boeck K, et al. Pseudomonas aeruginosa in the home environment of newly infected cystic fibrosis patients. Eur Respir J 2008; 31: 822-829.

42. Heirali A, McKeon S, Purighalla S, et al. Assessment of the microbial constituents of the home environment of individuals with cystic fibrosis (CF) and their association with lower airways infections. PLoS One 2016; 11: e0148534.

43. Zupančič J, Novak Babič M, Zalar P, et al. The black yeast Exophiala dermatitidis and other selected opportunistic human fungal pathogens spread from dishwashers to kitchens. PLoS One 2016; 11: e0148166.

44. Cayston. Cleaning and disinfecting. https://www.cayston. com/dosing/cleaning-and-disinfecting.

45. Caskey S, Stirling J, Moore JE, et al. Occurrence of Pseudomonas aeruginosa in waters: implications for patients with cystic fibrosis (CF). Lett Appl Microbiol 2018; 66: 537-541.

46. Moore JE, Thompson I, Crowe M, et al. Burkholderia cepacia from a sink drain. J Hosp Infect 2002; 50: 235-237.

47. Woodhouse R, Peckham DG, Conway SP, et al. Water filters can prevent Stenotrophomonas maltophilia contamination of nebuliser equipment used by people with cystic fibrosis. J Hosp Infect 2008; 68: 371-372

48. Blanc DS, Gomes Magalhaes B, Abdelbary M, et al. Hand soap contamination by Pseudomonas aeruginosa in a tertiary care hospital: no evidence of impact on patients. J Hosp Infect 2016 ; 93: 63-67.

49. Zimakoff J, Høiby N, Rosendal K, et al. Epidemiology of Pseudomonas aeruginosa infection and the role of contamination of the environment in a cystic fibrosis clinic. J Hosp Infect 1983; 4: 31-40.

50. Caetano JA, Lima MA, Di Ciero Miranda M, et al. Identification of bacterial contamination in liquid soap for hospital use. Rev Esc Enferm USP 2011; 45: 153-160. 
51. Döring G, Bareth H, Gairing A, et al. Genotyping of Pseudomonas aeruginosa sputum and stool isolates from cystic fibrosis patients: evidence for intestinal colonization and spreading into toilets. Epidemiol Infect 1989; 103: 555-564.

52. Reychler G, Aarab K, Van Ossel C, et al. In vitro evaluation of efficacy of 5 methods of disinfection on mouthpieces and facemasks contaminated by strains of cystic fibrosis patients. J Cyst Fibros 2005; 4: 183-187.

53. Jadhav S, Sahasrabudhe T, Kalley V, et al. The microbia colonization profile of respiratory devices and the significance of the role of disinfection: a blinded study. J Clin Diagn Res 2013; 7: 1021-1026

54. Caskey S, Moore JE, Rendall JC. In vitro activity of seven hospital biocides against Mycobacterium abscessus: Implications for patients with cystic fibrosis. Int J Mycobacterio/ 2018; 7: 45-47.

55. Collins MS, O'Brien M, Schramm CM, et al. Repeated hot water and steam disinfection of Pari LC Plus ${ }^{\circledR}$ nebulizers alter nebulizer output. J Cyst Fibros 2019; 18: 233-235.

56. Reychler G, Leonard A, Van Ossel C, et al. Impact of hypochlorite-based disinfection on bacterial contamination of cystic fibrosis patients' home-nebulisers. J Hosp Infect 2009; 72: 351-357.

57. Towle D, Baker V, Schramm C, et al. Ozone disinfection of home nebulizers effectively kills common cystic fibrosis bacteria pathogens. Pediatr Pulmonol 2018; 53: 599-604.

58. Djouhri-Bouktab L, Alhanout K, Andrieu V, et al. Soluble squalamine tablets for the rapid disinfection of home nebulizers of cystic fibrosis patients. J Cyst Fibros 2012; 11 555-559.

59. Millar BC, Bell J. Comment on "repeated hot water and steam disinfection of pari LC Plus ${ }^{\circledR}$ nebulizers alter nebulizer output". J Cyst Fibros 2019; 18: e16.

60. Towle D, Callan DA, Farrel PA, et al. Baby bottle steam sterilizers disinfect home nebulizers inoculated with bacterial respiratory pathogens. J Cyst Fibros 2013; 12: 512-516.

61. Towle D, Callan DA, Lamprea C, et al. Baby bottle steam sterilizers for disinfecting home nebulizers inoculated with non-tuberculous mycobacteria. J Hosp Infect 2016; 92 222-225.

62. Hohenwarter K, Prammer W, Aichinger W, et al. An evaluation of different steam disinfection protocols for cystic fibrosis nebulizers. J Cyst Fibros 2016; 15: 78-84.

63. Wabi Baby. Sterilization and drying should always go hand in hand. https://wabibaby.zendesk.com/hc/en-us/article attachments/115001439312/Test-Article.pdf.
64. Bakuridze L, Andrieu V, Dupont C, et al. Does repeated disinfection of the e-Flow rapid nebulizer affect in vitro performance? J Cyst Fibros 2007; 6: 309-310.

65. Rosaspina S, Salvatorelli G, Anzanel D. The bactericidal effect of microwaves on Mycobacterium bovis dried on scalpel blades. J Hosp Infect 1994; 26: 45-50.

66. Rosaspina S, Salvatorelli G, Anzanel D, et al. Effect of microwave radiation on Candida albicans. Microbios 1994 78: 55-59.

67. Sanborn MR, Wan SK, Bulard R. Microwave sterilization of plastic tissue culture vessels for reuse. Appl Environ Microbiol 1982; 44: 960-964

68. Le Brun PPH, Brimicombe RW, van Doorne $\mathrm{H}$, et al. The cleaning and disinfection of nebulizers used at home and in a cystic fibrosis centre. Eur Hosp Pharm 2000; 6: 58-63.

69. Pari. Cleaning and maintenance. https://www.pari.com/ us-en/education-ceu/cleaning-maintenance/.

70. Potts M. Desiccation tolerance of prokaryotes. Microbiol Rev 1994; 58: 755-805.

71. Moore JE, Moore RE, Bell J, et al. Importance of nebulizer drying for patients with cystic fibrosis. Respir Care 2020: respcare.07362

72. Moore JE, Millar BC. Susceptibility of the Mycobacterium abscessus complex to drying: implications for nebulizer hygiene in patients with cystic fibrosis. Int J Mycobacteriol 2020; 9: 173-175.

73. Moore JE, Millar BC. Susceptibility of Staphylococcus aureus (MSSA and MRSA) to drying: implications for nebulizer hygiene in patients with cystic fibrosis. J Hosp Infect 2020; 105: 366-368.

74. Alexander L, Carson J, McCaughan J, et al. Thinking inside the box - nebuliser care, safe storage and risk of infection. J Bras Pneumol 2020; 46: e20190226.

75. Rohan JM, Drotar D, Perry AR, et al. Training health care providers to conduct adherence promotion in pediatric settings: an example with pediatric asthma. Clin Pract Pediatr Psychol 2013; 1: 314-325.

76. Luby SP, Agboatwalla M, Feikin D, et al. Effect of handwashing on child health: a randomized controlled trial. Lancet 2005 366: 225-233.

77. Bowen A, Agboatwalla M, Ayers T, et al. Sustained Improvements in Handwashing Indicators more than 5 years after a cluster-randomised, community-based trial of handwashing promotion in Karachi, Pakistan. Trop Med Int Health 2013; 18: 259-267. 